\title{
La formación del profesional de la información en Venezuela: Aproximación histórico-analítica
}

\author{
Juan Carlos Linárez Pérez \\ Oficina de Planificación del Sector Universitario - OPSU, República Bolivariana de Venezuela
}

REVIEW

\begin{abstract}
Resumen
Analiza algunos elementos históricos anteriores a la implementación de los programas académicos para la formación de profesionales de la información en Venezuela. Estudia el proceso evolutivo mostrando las instituciones universitarias responsables de los planes de estudio, las iniciativas gubernamentales para aumentar la oferta académica, los tipos de cursos ofertados (técnico, pregrado, y postgrado), los títulos concedidos, algunos elementos de orientación de la actividad académica, y el enfoque de los planes de estudio actuales.
\end{abstract}

Palabras clave

Ciencias de la Información ; Formación profesional ; Currículo ; Universidades ; Venezuela

\section{The formation of the information professional in Venezuela: historical-analytical approach}

\begin{abstract}
Discusses some previous historical elements to the implementation of academic programs for the training of information professionals in Venezuela. Studies the evolutionary process, showing the university institutions responsible for curricula, the government initiatives to increase the academic offer, the types of offered courses (technical, undergraduate and postgraduate), the qualifications awarded, some elements of guidance for the scholar activity, and the current focus of its curriculum.
\end{abstract}

Keywords

Information science ; Professional training ; Curriculum ; Universities ; Venezuela

\section{Introducción}

A pesar de la llegada tardía de la imprenta a Venezuela -año de 1808, imprenta de Gallagher \& Lamb- la actividad bibliotecaria se inició prácticamente con la introducción de las primeras órdenes religiosas en lo que hoy es Venezuela. Franciscanos, Capuchinos, Dominicos, Agustinos y Jesuitas trajeron en sus equipajes una importante cantidad de textos litúrgicos (misales, breviarios, biblias y santorales) lo mismo que textos filosóficos y teologales que sirvieron para la constitución de las primeras bibliotecas eclesiales. Estas bibliotecas eran de uso exclusivo de la comunidad misionera, con lo cual la actividad bibliotecaria, es por así decirlo, incipiente en estos primeros años. Lo mismo sucedería con bibliotecas privadas propiedad de algunos funcionarios del régimen de la colonia así como a sacerdotes seculares, obispos en su mayoría, que atesoraban textos clásicos en sus casas de habitación o en los despachos gubernamentales. ${ }^{1}$

Para el año de 1810, Juan Germán Roscio, prócer de la independencia de Venezuela y secretario de Estado, firma el decreto de creación de la primera biblioteca pública de Caracas, que contemplaba el carácter amplio e incluyente de esta biblioteca: 
domingos, días festivos, y jueves. Nadie será admitido con capa y a todos se suministrará

tintero, pluma y papel, para extractos, ó apuntes. [... $]^{1}$

Sin embargo, la materialización del mandato de creación no se produciría sino hasta el 13 de junio de 1833 fecha en la cual se crea, ya no la Biblioteca Pública de Caracas, sino la Biblioteca Nacional de Venezuela. Esta fecha marca el inicio formal de la actividad bibliotecaria pública en nuestro país y para este momento se designó como primeros bibliotecarios a Diego Bautista Urbaneja y Antonio Leocadio Guzmán, ambos ministros del presidente en funciones, general José Antonio Páez. ${ }^{1}$

Como es de esperar, estos primeros bibliotecarios si bien eran personas de reconocida labor intelectual y cultural, no poseían ninguna formación bibliotecaria salvo aquella que les había deparado la relación estrecha con los libros e impresos.

A partir de la segunda mitad del siglo XIX y hasta mediados del siglo XX, la formación de bibliotecarios y archiveros en Venezuela se llevó a cabo en las mismas organizaciones de información; este hecho resulta similar y recurrente en todo el mundo, es decir, la formación de profesionales de la información se llevó a cabo en sus inicios en las bibliotecas, archivos y museos.

Otro aspecto que resulta muy revelador y da cuenta del alto compromiso de los primeros bibliotecarios públicos en Venezuela son los valores humanísticos, el alto bagaje cultural e intelectual y el compromiso social. Personajes como José María Vargas, primer presidente civil en Venezuela y bibliotecario entre 1836 y 1858 ; el historiador Juan Vicente González, en el periodo 1858-1863; el científico Adolfo Ernst, en el periodo 1876-1889 y se suman a los ya mencionados, Diego Bautista Urbaneja y José Leocadio Guzmán, son los precursores de la Biblioteca Nacional de Venezuela en el periodo comprendido entre 1833 y 1835, como la pléyade de ilustres bibliotecarios que prestaron un encomiable servicio y transmitieron sus experiencias y saberes a un importante número de personas que destinaron su vida y vocación al servicio en las bibliotecas y archivos de la Venezuela de finales del siglo $X I X^{1}$. En la primera mitad del siglo $X X$, la situación de la formación de bibliotecarios y archiveros fue similar a la antecedente, al igual que la calidad intelectual y la probidad ética de los directores de la Biblioteca Nacional de Venezuela, por ende responsables de la política de bibliotecas públicas en nuestro país. Nombres como Rafael Requena, reconocido pintor y humanista, Manuel Segundo Sánchez, insigne historiador y bibliógrafo, el historiador Caracciolo Parra León y el escritor Luis Urbaneja Achepohl, precursor del criollismo como género de la literatura venezolana, son sólo algunos de los directores-bibliotecarios que ofrendaron sus esfuerzos en pro de la actividad bibliotecaria venezolana.

Será hasta casi el inicio de la segunda mitad del siglo XX cuando se establece formalmente la formación de profesionales de la información en el área bibliotecaria, hecho acaecido en la Universidad Central de Venezuela a través de la creación de los estudios técnicos para bibliotecónomos. Sobre este aspecto, cabe resaltar que estos primeros bibliotecarios, formados académicamente, eran personas con experiencia en la actividad, en su mayoría provenían de bibliotecas públicas y universitarias, hecho que facilitó enormemente el desarrollo de las actividades de formación escolarizada. También resalta el celo con el cual las universidades, Central de Venezuela y del Zulia, monopolizaron la formación de profesionales de la información en nuestro país por casi cincuenta años. Sobre este particular, se llegó a subestimar la calidad de la formación técnica que se había iniciado en 1997 y que egresó la primera cohorte de técnicos superiores universitarios en el año 2000, desconociendo el valor académico de la misma y, por ende, la posibilidad de los egresados de proseguir estudios a nivel de licenciatura y postgrado. Por más de seis años se extendió esta imposibilidad hasta la concreción de convenios internacionales que posibilitarían una apertura y una ruptura paradigmática en la formación de profesionales de la información en Venezuela.

\section{Formación de profesionales de la información en Venezuela}

En Venezuela, la formación de profesionales de la información, especialmente en el área de bibliotecas y archivos fue, desde su inicio en 1948 hasta 1996, gestión exclusiva de dos de las principales universidades del país; la Universidad Central de Venezuela y la Universidad del Zulia, en ambos casos a partir de la creación de la Escuela de Bibliotecología y Archivología. En 1997, la oferta académica en el área informacional se amplió con la creación de un programa de formación técnica-universitaria en el antiguo Instituto Universitario Experimental de Tecnología Andrés Eloy Blanco ubicado en la ciudad de Barquisimeto en el Estado Lara. También, en esta entidad federal una universidad privada, Universidad Yacambú, se propuso la creación de estudios a nivel de licenciatura en Información y Documentación. 
Otra iniciativa que ha obtenido mucha acogida en la zona central del país es la oferta de la Universidad de Carabobo, a través de la Fundación Centro de Información y Documentación, FUNDACID, de formar a nivel de técnico medio Analistas Documentalistas y Asistentes de Biblioteca; también, en esta zona del país la Universidad Pedagógica Experimental Libertador oferta a través del Instituto Pedagógico Rural El Mácaro, ubicado en la población del mismo nombre en el Estado Aragua, dos diplomados el de Bibliotecología y Ciencias de la Información y de Bibliotecas Escolares.

En lo referido a la formación de museólogos en nuestro país, cabe destacar, que hasta la fecha, las ofertas académicas para cursar estudios en este campo del conocimiento científico se focalizan solamente a nivel de especialización y maestría. Igualmente, destaca que estas ofertas académicas focalizan sus esfuerzos en los aspectos estéticos y museográficos del campo de conocimiento.

A nivel de postgrado, en el campo general de las Ciencias de la Información, las ofertas de estudio están situadas en especializaciones y maestrías, careciendo hasta la fecha de un programa de estudios doctorales.

Seguidamente analizaremos de acuerdo a la institución responsable de su administración y titulación cada una de las ofertas académicas en el área informacional en Venezuela.

\subsection{Universidad Central de Venezuela}

No sólo es la primera universidad creada en nuestro país sino también la primera casa de estudios universitarios en incorporar en su oferta académica formación en el área informacional.

Para el año de 1948 el Consejo Universitario de la UCV resuelve la creación, a través de la Facultad de Filosofía y Letras, de una oferta académica para la formación de Técnicos en Biblioteconomía en dos años, ello a objeto de atender necesidades de formación en el área bibliotecaria de un importante número de personas que laboraban en bibliotecas públicas y académicas. Posteriormente, en el año de 1956, se incorporan temáticas relacionadas con el área del archivo. ${ }^{2}$

A partir de 1960 las autoridades de la universidad resuelven trasformar los estudios técnicos en estudios de licenciatura en Bibliotecología y Archivología así como adscribir la Escuela de Bibliotecología y Archivología a la Facultad de Humanidades y Educación para lo cual se amplió el plan de estudios a cuatro años. Posteriormente, en 1970 se trasformó en un plan de estudios de cinco años para titular separadamente licenciados en Bibliotecología y Archivología, este plan de estudios prevé un ciclo básico conjunto de cuatro semestres y dos ciclos profesionales de seis semestres. ${ }^{2}$

En cuanto a la oferta académica para la prosecución de estudios a nivel de postgrado existe especialización y maestría denominada Información y Comunicación para el Desarrollo²; por su parte, la Facultad de Arquitectura y Urbanismo de esta universidad oferta una especialización en Museología que, de acuerdo al perfil de ingreso, está orientada más a los aspectos museográficos que a los documentales de este campo el conocimiento científico.

\subsection{Universidad del Zulia}

Los estudios en Bibliotecología y Archivología fueron creados en esta universidad en el año de $1962^{3}$, inicialmente adscritos a la Escuela de Letras, conferían el título de Licenciado en Letras Mención Bibliotecología y Archivología. A finales de la década de los ochenta del siglo XX, las autoridades universitarias acordaron la creación de la Escuela de Bibliotecología y Archivología de la Universidad del Zulia (EBA-LUZ) y su adscripción a la Facultad de Humanidades y Educación, desde entonces se confiere el título de Licenciado en Bibliotecología y Archivología basado en una visión integral y holística del currículo ${ }^{4}$, sin embargo, esta declaratoria holística no exime al diseño curricular de un abordaje meramente disciplinar, el cual se refleja explícitamente en el plan de estudios de esta universidad $^{5}$. En lo referido a estudios de postgrado, EBA-LUZ oferta la Maestría en Ciencias de la Información Mención Gerencia del Conocimiento.

Los objetivos que se propone la EBA-LUZ para la formación de profesionales de la información se concentran en el desarrollo de competencias que le permitan a éstos el establecimiento de políticas, la elaboración de planes, programas y proyectos a objeto de suministrar y diseminar información, también, la planificación y dirección de unidades de información en lo concerniente a la organización del 
conocimiento, determinación de necesidades de información de los usuarios y la vinculación de las unidades de información con la realidad social y organizacional del país. ${ }^{3}$

\subsection{Instituto Universitario Experimental de Tecnología Andrés Eloy Blanco/Universidad Politécnica Territorial de Lara Andrés Eloy Blanco}

Desde 1997, el antiguo Instituto Universitario Experimental de Tecnología Andrés Eloy Blanco administraba regularmente un programa de formación académica técnica-universitaria, en una especialidad que guarda estrecha relación con las ciencias de la información: T.S.U. en Información y Documentación; la experiencia acumulada en el ejercicio docente y administrativo sirvió para plantear una evolución en materia curricular y en la titulación de los estudiantes.

La formación académica de este profesional de la información se orientaba hacia la consolidación de un conjunto de tareas ocupacionales que serían ejecutadas en las organizaciones de información donde le correspondía desenvolverse laboralmente y culminaba su formación con una práctica profesional denominada pasantía que servía para la puesta en práctica de los conocimientos, habilidades y destrezas desarrollados en la formación académica previa. ${ }^{6}$

Para finales del año 2007, ante el empuje que venía ejerciendo La Revolución Bolivariana en materia educacional, el Departamento de Información y Documentación comisionó a un grupo de docentes para la preparación del PNF en Ciencias de la Información, así como, de un programa municipalizado con el objeto de llevar formación académica universitaria a personas que habitan en municipios foráneos a la ciudad de Barquisimeto.

Más de un año y medio de reuniones, debates y revisiones de otros planes de estudios conexos al área del conocimiento de las Ciencias de la Información, dieron como resultado un documento de tipo técnico-curricular que plantea la formación de profesionales de la información desde una visión integradora de las disciplinas que declaran explícita o implícitamente su interés por estudiar e interpretar el fenómeno informacional contemporáneo. Destaca, además, la visión del proyecto sociointegrador como elemento que consolida la formación profesional desde la perspectiva práctica e involucra al participante desde el inicio hasta el final en la dinámica laboral y comunitaria. ${ }^{7}$

\subsection{Universidad Nacional Experimental Francisco de Miranda}

Esta institución ubicada en la ciudad de Coro, en el Estado Falcón, administra la Maestría en Museología. La oferta académica se coordina a través del área de turismo de la mencionada institución y está dirigida a profesionales que laboran en instituciones museísticas y los aspectos más representativos de esta maestría se orientan hacia los aspectos museográficos, es decir, hacia los aspectos técnicos-operativos de la actividad museística. ${ }^{8}$

\subsection{Universidad Yacambú}

Esta institución universitaria de carácter privado cuenta, en su oferta académica, con la Licenciatura en Información y Documentación, esta carrera-programa se propone la formación de profesionales, según lo refiere la página web institucional:
[...] Para la generación, recolección, organización, almacenamiento, recuperación, interpretación, difusión y uso de la información en el contexto de una sociedad global que vive un proceso de transición paradigmática; con una sólida base teórica, metodológica y práctica en el campo de la ciencia de la información para desempeñarse como profesional de procesos técnicos, analista, asesor, gerente e investigador, que facilite el acceso a la información con un elevado sentido de la eficiencia y eficacia. ${ }^{9}$

El plan de estudios, vigente desde el año de 2009, contempla el estudio de los fundamentos teóricosconceptuales de Bibliotecología, Archivología y Ciencia de la Información, así como, aspectos relacionados con la organización del conocimiento, gestión de información y uso de tecnologías, también, gerencia, investigación y comportamiento organizacional. 
A la fecha, esta institución no oferta ningún curso a nivel de postgrado en el área de las Ciencias de la Información.

\subsection{Universidad José Antonio Páez}

También de carácter privado, esta institución radicada en la ciudad de Valencia en el Estado Carabobo, oferta una maestría denominada Gerencia de Tecnología de la Información, la cual fue aprobada por resolución del Consejo Nacional de Universidades, ente adscrito al Ministerio del Poder Popular para la Educación Universitaria. La fundamentación de esta maestría refiere a la importancia que la sociedad ha conferido a la información en las últimas décadas así como el carácter estratégico y operacional de la misma. Los objetivos que se propone esta maestría están vinculados con desarrollo de competencias que le permitan a los egresados contribuir activamente en la resolución, por la vía del conocimiento, de problemáticas inherentes a la existencia de conglomerados sociales y organizaciones sociales en su más amplio espectro. ${ }^{10}$

\subsection{Universidad de Carabobo}

Esta institución oferta, a través de la Fundación Centro de Información y Documentación (FUNDACID), unidad adscrita a la Dirección General de Biblioteca Central Universidad de Carabobo, cursos a nivel de técnico medio en el área bibliotecaria. Estos cursos tienen un costo que debe ser cancelado por los interesados.

El curso de Analista Documentalista tiene por objeto la formación de recurso humano para el tratamiento de información científico-tecnológica y está orientado para personas que trabajan en centros de documentación de universidades y centros de investigación. Los contenidos que se tratan en este curso se focalizan en los aspectos técnicos operativos que rigen este tipo de organizaciones, entre ellos destacan: tratamiento de la información en lo referido a su organización y representación, uso y manejo de las tecnologías de información digital, servicios de información y usuarios.

Por su parte, el curso Asistente de Biblioteca, está orientado hacia la formación bibliotecaria en aspectos meramente técnicos, siendo su objeto principal la capacitación de personal auxiliar en catalogación y clasificación, búsqueda y recuperación de información, así como, la atención de usuarios. Cabe destacar, que estos cursos tienen muy buena acogida ya que representan una alternativa de formación para personas que laboran en organizaciones de información circunvecinas a la Universidad de Carabobo, específicamente en los estados Aragua y Cojedes. ${ }^{11}$

\subsection{Universidad Pedagógica Experimental Libertador}

Esta casa de estudios universitarios tiene la responsabilidad de formar al magisterio venezolano; en sus nueve sedes, repartidas a nivel nacional, se forma un importante número de docentes en carreras de pre y postgrado, lo mismo que la profesionalización de docentes en ejercicio en el denominado Instituto Pedagógico Rural ubicado en la población de El Mácaro en el Estado Aragua. Es en este último instituto que se ofertan los diplomados en Bibliotecología y Ciencias de la Información y de Bibliotecas Escolares $^{12}$. Estos cursos de doscientas horas académicas cada uno, están orientados a formar docentes en ejercicio en los aspectos relacionados con la actividad bibliotecaria, para desempeñarse en bibliotecas escolares como en centros de información y documentación de instituciones científicas dependientes del Ministerio del Poder Popular para la Educación. En ambos casos, los interesados deben cancelar el costo de la matrícula, es decir, no son gratuitos.

\subsection{Universidad Simón Bolívar}

Esta prestigiosa universidad venezolana conformó a mediados de la década de los ochenta y hasta principios de los noventa del siglo XX lo que sería el primer curso de postgrado en el área informacional. Esta iniciativa estuvo a cargo del insigne investigador y docente venezolano Iraset Páez Urdaneta quien, en 1984 creó la especialización en Servicios de Información. Para la fecha el profesor 
Páez venía de desempeñarse exitosamente como director de la Unidad de Investigación de la Biblioteca Nacional de Venezuela y la experiencia acumulada en sus años de bibliotecario-investigador más el bagaje académico que poseía, sirvieron para estructurar esta oferta académica ${ }^{13}$. Consecuencia de su temprana desaparición física en 1994 la filosofía organizacional de la especialización se trasladó hacia el área Informática y perdió todo vínculo con el campo de conocimiento que exploran las Ciencias de la Información.

Otro aspecto de muy especial significación en la formación de profesionales de la información en Venezuela lo representa, sin duda alguna, la firma de convenios educativos con la República de Cuba. En el contexto de los convenios binacionales que adelantan ambos gobiernos desde 1999 se acordó, en el año de 2006, la puesta en marcha de un programa especial de homologación de técnicos superiores universitarios en Información y Documentación para su acreditación como licenciados en Bibliotecología y Ciencias de la Información. Para este cometido, representantes del Convenio Binacional Venezuela-Cuba designaron al Departamento de Bibliotecología y Ciencia de la Información, unidad académica dependiente de la Facultad de Comunicaciones de la Universidad de La Habana, para llevar a cabo un diagnóstico que permitiera conocer de primera mano la realidad venezolana en lo referente a formación de profesionales de la información.

Con el objeto de aumentar la calificación académica de un grupo de profesionales que venían desempeñándose como auxiliares de docencia en el antiguo Instituto Universitario Experimental de Tecnología Andrés Eloy Blanco en la ciudad de Barquisimeto y en el programa municipalizado en Información y Documentación; se llevó a cabo esta homologación gracias a los esfuerzos de los docentes de la Universidad de La Habana, quienes no sólo planificaron curricular, logística y operacionalmente los cursos de homologación sino que titularon a los participantes. La homologación fue estructurada a través de la planificación de doce asignaturas que abordaban temáticas relacionadas con las Ciencias de la Información, el objetivo estaba claro; analizar y valorar aspectos teóricos conceptuales del campo de conocimiento que no habían sido tratados en los estudios técnicos; reforzar aspectos relacionados con el rol social e histórico del profesional de la información; presentar métodos de abordaje para el desempeño en servicios de información; analizar la nueva visión de la Organización de la Información y el Conocimiento de cara a la realidad que dibuja el desarrollo y masificación de las tecnologías de información digital en los entornos informacionales especialmente en las organizaciones de información y valorar la importancia de la actividad informacional en el desarrollo de la sociedad.

En el año de 2009, un grupo de treinta y ocho técnicos recibieron su titulación como licenciados ${ }^{14}$ después de cumplir con dos años de formación presencial y un trabajo especial de grado. Estos profesionales están ahora cumpliendo tareas docentes en la universidad y en el programa municipalizado que adelanta la Misión Sucre en varios municipios del Estado Lara, este programa municipalizado se extendió a otras regiones del país: Carabobo, Mérida, Sucre y Miranda.

Adicionalmente, en el año 2010, se dio inicio a un curso de postgrado a nivel de maestría que fue extendido al Estado Carabobo y al Distrito Capital, este último funciona en las instalaciones de la Biblioteca Nacional; en este contexto cursan estudios de Maestría en Ciencias de la Información un grupo de sesenta maestrantes. También, se prevé la conformación de un curso especial de doctorado que debe iniciar en el año de 2014, este hecho marcaría el inicio de estudios doctorales en el campo de las Ciencias de la Información en nuestro país lo cual tributaría al fortalecimiento de la investigación y docencia del área.

Finalmente, podemos reseñar la existencia de dos iniciativas de instaurar estudios informacionales en dos universidades de carácter nacional, nos referimos a la Universidad Bolivariana de Venezuela y la Universidad Nacional Abierta, sin embargo, a la fecha no han sido autorizadas por el ente ministerial en materia de educación universitaria.

Seguidamente se presenta, a modo ilustrativo, una cuadro-resumen de la distribución por universidades de los estudios informacionales en Venezuela, también, se reseña la titulación que confieren las instituciones universitarias así como el carácter público o privado de las mismas (véase Cuadro 1). 


\begin{tabular}{|c|c|c|c|c|}
\hline Institución universitaria & Carácter & $\begin{array}{c}\text { Zona } \\
\text { Geográfica }\end{array}$ & Tipo de Curso & Titulación \\
\hline $\begin{array}{l}\text { Universidad Central de } \\
\text { Venezuela }\end{array}$ & Público & $\begin{array}{l}\text { Centro, } \\
\text { Distrito } \\
\text { Capital }\end{array}$ & $\begin{array}{c}\text { Pre y } \\
\text { postgrado }\end{array}$ & $\begin{array}{l}\text {-icenciado en Bibliotecología. } \\
\text {-icenciado en Archivología. } \\
\text { Maestría en Información y Comunicación } \\
\text { para el Desarrollo. *Especialización en } \\
\text { Museología. }\end{array}$ \\
\hline Universidad del Zulia & Público & $\begin{array}{l}\text { Occidente } \\
\text { Zulia }\end{array}$ & $\begin{array}{c}\text { Pre y } \\
\text { postgrado }\end{array}$ & $\begin{array}{l}\text { *Licenciado en Bibliotecología y } \\
\text { Archivología. } \\
\text { *Maestría en Ciencias de la Información } \\
\text { Mención Gerencia del Conocimiento. }\end{array}$ \\
\hline $\begin{array}{l}\text { Universidad Politécnica } \\
\text { Territorial de Lara Andrés } \\
\text { Eloy Blanco }\end{array}$ & Público & $\begin{array}{l}\text { Centro- } \\
\text { Occidente, } \\
\text { Lara }\end{array}$ & Pregrado & $\begin{array}{l}\text { *Licenciado en Ciencias de la Información. } \\
\text { *Técnico Superior Universitario en } \\
\text { Información y Documentación. }\end{array}$ \\
\hline Universidad Yacambú & Privado & $\begin{array}{l}\text { Centro- } \\
\text { Occidente, } \\
\text { Lara }\end{array}$ & Pregrado & $\begin{array}{l}\text { *Licenciado en Información y } \\
\text { Documentación. }\end{array}$ \\
\hline $\begin{array}{l}\text { Universidad José Antonio } \\
\text { Páez }\end{array}$ & Privado & $\begin{array}{l}\text { Centro, } \\
\text { Carabobo }\end{array}$ & Postgrado & $\begin{array}{l}\text { *Maestría en Gerencia de Tecnología de la } \\
\text { Información. }\end{array}$ \\
\hline $\begin{array}{l}\text { Universidad Nacional } \\
\text { Experimental Francisco } \\
\text { de Miranda }\end{array}$ & Público & $\begin{array}{l}\text { Occidente, } \\
\text { Falcón }\end{array}$ & Postgrado & *Maestría en Museología. \\
\hline Universidad de Carabobo & Público & $\begin{array}{l}\text { Centro, } \\
\text { Carabobo }\end{array}$ & $\begin{array}{l}\text { Técnico } \\
\text { Medio }\end{array}$ & $\begin{array}{l}\text { *Analista Documentalista. } \\
{ }^{*} \text { Asistente de Biblioteca. }\end{array}$ \\
\hline $\begin{array}{l}\text { Universidad Pedagógica } \\
\text { Experimental Libertador }\end{array}$ & Público & $\begin{array}{l}\text { Centro, } \\
\text { Aragua }\end{array}$ & Diplomado & $\begin{array}{l}\text { *Diplomado en Bibliotecas Escolares } \\
\text { *Diplomado en Bibliotecología y Ciencias } \\
\text { de la Información. }\end{array}$ \\
\hline
\end{tabular}

Cuadro 1: Instituciones, distribución geográfica, tipo de curso y titulaciones.

Como es posible advertir, la formación académica de los profesionales de la información en Venezuela se concentra en el centro del país (Distrito Capital, estados Aragua y Carabobo) y en el occidente (estados Lara, Falcón y Zulia); este hecho resulta una gran limitación para las personas interesadas en cursar estudios informacionales que habitan la región oriental, los llanos y el sur del país.

Otro aspecto resaltante de esta tabla resumen lo representan la diversidad de titulaciones que confieren las instituciones responsables de la formación de profesionales de la información en Venezuela.

En el caso de la Universidad Central de Venezuela se confieren tres titulaciones: Dos corresponden a estudios de pregrado (Licenciado en Bibliotecología y Licenciado en Archivología) y una corresponde a postgrado (Magíster en Información y Comunicación para el Desarrollo); la Universidad del Zulia confiere el título integral en Bibliotecología y Archivología y el Magister Scenciarum en Ciencias de la Información Mención Gerencia del Conocimiento; la Universidad Politécnica Territorial de Lara Andrés Eloy Blanco confiere los títulos de Licenciado en Ciencias de la Información y como salida intermedia el de Técnico Superior Universitario en Información y Documentación; la Universidad Yacambú confiere el título de Licenciado en Información y Documentación; la Universidad José Antonio Páez confiere el título de Magíster en Gerencia de Tecnologías de la Información; la Universidad Nacional Experimental Francisco de Miranda el título de Magíster en Museología y las Universidades de Carabobo y Pedagógica Experimental Libertador certifican sus cursos y diplomados con las denominaciones de Analista Documentalista, Asistente de Bibliotecas, Bibliotecología y Ciencias de la Información y Bibliotecas Escolares.

\section{Los diseños curriculares delos programas}

En Venezuela la formación de profesionales de la información obedeció desde 1948, año en el que se aprueba el primer plan de estudios para la formación de bibliotecónomos, hasta 2008, año en el que se instaura el Programa Nacional de Formación en Ciencias de la Información, a una visión eminentemente disciplinar, es decir, la formación y titulación se lleva a cabo atendiendo puntualmente, bien sea, la actividad bibliotecaria (Bibliotecología) o la actividad archivística (Archivología). Si bien la Universidad del Zulia confiere el título de 
Licenciado en Bibliotecología y Archivología, la estructura curricular está diseñada para explorar separadamente el área de biblioteca y el área de archivo. Otras experiencias como la de la Universidad Yacambú, centra su esfuerzo curricular en el área documental. Un planteamiento distinto, al menos en lo que conceptualmente refiere y en la titulación, es la iniciativa adoptada por la Universidad Politécnica Territorial de Lara Andrés Eloy Blanco en su Programa Nacional de Formación en Ciencias de la Información, sin embargo, analizando el diseño curricular advertimos que la visión disciplinar persiste, sobre todo, en el abordaje temático de las unidades curriculares (asignaturas) que este diseño curricular contempla.

Seguidamente se presentan y analizan los planes de estudio correspondientes a los estudios informacionales en Venezuela.

\subsection{Universidad Central de Venezuela}

Ser la primera casa de estudios universitarios en Venezuela en instaurar formación en el campo informacional, le confiere una dilatada experiencia en los aspectos tecno-curriculares. En el año 1948, con la aprobación que hiciera el Consejo Universitario de esta casa de estudios, se concretó un plan de estudios de quince asignaturas para la formación de Técnico en Biblioteconomía y centró sus esfuerzos en la formación práctica, de allí que se hiciera hincapié en los aspectos catalográficos y clasificatorios de las colecciones bibliotecarias sin atender aspectos archivísticos. Este plan de estudios estuvo vigente sólo para la primera cohorte que egresó en 1950; a partir de este año se incrementó a diecisiete asignaturas y a tres años la formación técnica. Durante esta década sólo se llevaron a cabo revisiones curriculares a objeto de incorporar asignaturas que a finales de la década eran veintiún y persistía la formación eminentemente bibliotecaria. A partir del año de 1960, se incorporan los estudios archivísticos, se incrementa a cuatro años la formación y comienzan las titulaciones como licenciados. Esta visión ocuparía toda la década de los sesenta; a partir del año 70 se establecen dos modalidades: Estudios diurnos con una duración de cuatro años y estudios nocturnos con una duración de cinco años. Para el año de 1978 se llevaría a cabo un cambio curricular que prácticamente sigue vigente hasta la fecha, con revisiones y cambios menores en 1982 y 2002, este cambio curricular estableció, entre otros elementos, la duración de la formación en cinco años distribuidos en diez semestres. Desde el año de 2005, la Escuela de Bibliotecología y Archivología de la UCV intenta la concreción de un nuevo diseño curricular que prevé la adopción de la visión holística del campo de conocimiento, así como, la denominación de Ciencias de la Información y el abordaje epistemológico de la interdisciplinariedad como basamento fundamental; sin embargo, a la fecha esa transformación curricular no se ha podido formalizar persistiendo la visión y el abordaje disciplinar y la titulación separada para bibliotecólogos y archivólogos. ${ }^{2}$

Otro aspecto que caracteriza los diseños curriculares implementados por esta casa de estudios lo representa la tendencia hacia la sobre-valoración de los elementos técnicos y prácticos de las actividades bibliotecarias y archivísticas sobre los aspectos teóricos conceptuales de estas disciplinas científicas.

\subsection{Universidad del Zulia}

Con la incorporación de los estudios informacionales en el año de 1962, la Escuela de Letras de esta institución daba un paso muy importante; regionalmente hablando sentaba las bases para formar en su propio seno profesionales en el campo informacional lo cual no sólo aseguraba a los jóvenes zulianos la posibilidad de cursar estudios en Bibliotecología y Archivología en su propia entidad federal, sino que también garantizaba que esta formación estaba consustanciada con la realidad zuliana.

El plan de estudios de 1962 y los cambios menores realizados en 1965 y 1970 estaban estrechamente vinculados a la formación en el campo de la letras; Historia, Literatura y Filosofía representaban una importante carga temática, de hecho, el título que se confería para este momento era el de Licenciado en Letras Mención Bibliotecología y Archivología; en el campo informacional propiamente dicho, la carga temática de estos planes de estudio estaba orientada hacia los aspectos prácticos de la profesión, predominando la formación en Organización de la Información bajo el esquema de la fisicalidad. Los siguientes once años, es decir, entre 1983 a 1994 representan el periodo de separación de los estudios informacionales de la Escuela de Letras y su auto definición como un campo del 
conocimiento independiente que demandaba una estructura propia, se crean cinco departamentos (Archivología, Bibliotecología, Gerencia de Información, Informática e Investigación y Documentación e Información) y se eleva a la condición de Escuela de Bibliotecología y Archivología. La trasformación del diseño curricular pasa por la incorporación de elementos gerenciales y tecnológicos al plan de estudios. ${ }^{5}$

En el año de 1995 se adopta una nueva visión tecno-curricular al incorporar como basamento curricular la formación en competencias desde una perspectiva holística ${ }^{4}$. Este plan de estudios con cambios menores sigue vigente en la actualidad y confiere el título de Licenciado en Bibliotecología y Archivología.

\subsection{Universidad Politécnica Territorial de Lara Andrés Eloy Blanco}

El Programa Nacional de Formación en Ciencias de la Información está fundamentado desde una visión abierta, flexible, transferible y multi-referencial del currículo, en este sentido, se propone la formación de profesionales de la información con principios éticos; comprometidos con el desarrollo del país, críticos, creativos; con vocación científica y sensibilidad social para investigar, diseñar y ejecutar proyectos informacionales, con bases teóricas y tecnológicas de alta calidad para el procesamiento, el análisis, la gestión y la difusión de la información y el conocimiento en las instituciones, organizaciones y comunidades; capaz de fortalecer la cultura informacional en las mismas a través del estímulo para el desarrollo de habilidades de información en los usuarios, aspecto que contribuye a la búsqueda de la equidad en el acceso a la misma. ${ }^{7}$

El periodo de formación es de cuatro años, cada uno de los cuales recibe la denominación de trayecto; cada trayecto contempla un número de unidades curriculares y la planificación y ejecución de un proyecto socio-integrador que tributa hacia la consolidación de competencias específicas del área profesional, así como, el fortalecimiento de valores sociales. Cada proyecto se circunscribe en una dimensión específica del campo de conocimiento, los mismos, deben ser planificados en entornos comunitarios y deben dar una respuesta a una situación problemática diagnosticada. Se debe tomar en cuenta, la valoración de las fuentes de información para conocer su utilidad social, técnica o científica, el valor probatorio, testimonial, histórico o estético, conforman el primer proyecto; posteriormente, el estudiante comprenderá la importancia y utilidad social de la organización de la información y el conocimiento, seguidamente, estudiará las necesidades de información de los usuarios para finalmente, comprender la importancia de la planificación, creación y administración de organizaciones o servicios de información. ${ }^{7}$

El proyecto socio-integrador se fortalece académica y metodológicamente con las unidades curriculares respectivas del trayecto y con un conjunto de talleres complementarios planificados en función de las realidades de cada grupo de estudiantes y de la naturaleza del proyecto que se está desarrollando; los aspectos relacionados con las tecnologías de la información digital son tratados en estos talleres lo cual garantiza una apropiación por parte del estudiantes de habilidades y destrezas para el uso y aplicación de estas tecnologías. ${ }^{7}$

El PNF en Ciencias de la Información, en consonancia con las líneas curriculares instruidas desde el Ministerio del Poder Popular para la Educación Universitaria, concretó la integración interinstitucional con los entes más estrechamente relacionados con el tema informacional en nuestro país; para este cometido las autoridades de la Universidad Politécnica Territorial de Lara Andrés Eloy Blanco llevaron a cabo la firma de un convenio de cooperación mutua con las autoridades del Instituto Autónomo Biblioteca Nacional y el Archivo General de la Nación, ello ha permitido entre otros logros la apertura de una aldea universitaria en los espacios de las instituciones antes mencionadas. Esta aldea emprendió la profesionalización de casi cien estudiantes en la primera cohorte, en su mayoría trabajadores de las instituciones involucradas en el convenio. También, destaca la conformación del Comité Interinstitucional del PNF en Ciencias de la Información, iniciativa del ministerio, que agrupa una representación de la academia y el sector empleador, así como de los entes rectores de las políticas informacionales de nuestro país; el objeto de este comité interinstitucional es la adecuación del diseño curricular a la realidad venezolana en lo que a organizaciones de información se refiere. También, analiza la pertinencia de los enunciados y contenidos curriculares con respecto a las políticas en 
materia de Educación Universitaria, además, de analizar la viabilidad de la instauración del programa nacional en otras universidades politécnicas del país.

\subsection{Universidad Yacambú}

La oferta académica de esta institución prevé la formación en cuatro años para modalidad presencial y cinco años para la modalidad a distancia, la distribución de las asignaturas se lleva a cabo por trimestres, conformando en total entre dieciséis y veinte trimestres en dependencia de la modalidad cursada. El plan de estudios correspondiente a la licenciatura en Información y Documentación está estructurado sobre la base humanística con componentes lingüísticos, históricos, gerenciales, tecnológicos y documentales; también, hace énfasis en aspectos técnicos-operativos de la dinámica bibliotecaria y archivística, así como, aspectos relacionados con la documentación en áreas específicas de la sociedad (Documentación Jurídica, Documentación Científica y Documentación Tecnológica). Este plan de estudios, vigente desde el año 2009, explora las Ciencias de la Información y todo su contexto, procesamiento de la información y el conocimiento, gestión de la información, tecnología de la información y de la comunicación, gestión de unidades, servicios y sistemas de información, gerencia, investigación, comportamiento y desarrollo profesional. ${ }^{9}$

\subsection{Universidad José Antonio Páez}

Los estudios de este postgrado denominado Maestría en Gerencia de Tecnología de la Información se llevan a cabo por el régimen de asignaturas, unidades de crédito y prelaciones de asignaturas tal como lo establecen las normas vigentes en la Universidad. Para acceder a las asignaturas del plan de estudios, el participante debe aprobar un curso introductorio obligatorio que consta de seis asignaturas de ocho horas académicas cada una. El plan de estudios tiene un total de once asignaturas que suman treinta y dos unidades de crédito, cada asignatura tendrá una duración que oscila entre treinta y dos y cuarenta y ocho horas académicas. De las once asignaturas, dos se clasifican como básicas y nueve como profesionales. El participante podrá concluir en dos años el plan de estudios. ${ }^{10}$

\subsection{Universidad Nacional Experimental Francisco de Miranda}

La oferta académica de la Maestría en Museología responde a un enfoque teórico-práctico centrado en el desarrollo de la persona como eje y centro del proceso educativo. Asimismo, toma en cuenta las características de adulto del participante al diseñar una metodología del aprendizaje con enfoque andragógico. Como fundamento básico de todo estudio de postgrado, la investigación se constituye en un eje curricular a lo largo de los módulos-asignaturas, previsto para su desarrollo, el cual culmina con la presentación de una tesis de grado sobre una de las áreas de la museología integrada a este plan de estudio. El plan de estudios está integrado además por un conjunto de módulos-asignaturas obligatorias que dan al participante una visión y una capacitación integral en museología que junto con la tesis perfilan al participante hacia las áreas de especialización ya mencionadas. La visión epistemológica de esta oferta de estudio se centra principalmente en aspectos museológicos y museográficos sin que la documentación museística aparezca como parte de los contenidos que se desarrollan en las asignaturas. ${ }^{8}$

\subsection{Universidad Pedagógica Experimental Libertador}

Los diplomados que oferta esta casa de estudios a través del Instituto Pedagógico Rural El Mácaro, está dirigida a docentes en ejercicio. En ambos casos son cursos de doscientas horas académicas y destaca el hecho, en el caso del Diplomado en Bibliotecología y Ciencias de la Información, que incorpora la Estadística, la formación de usuarios como elementos presentes en los ejes temáticos. ${ }^{12}$ 


\subsection{Universidad de Carabobo}

Los cursos ofertados por esta universidad a través de la Fundación Centro de Información y Documentación, tienen una duración de ciento sesenta y cuatro horas académicas cada uno, los ejes temáticos prestan mucha atención a los procesos técnicos de la información y están diseñados para la formación de asistentes en organizaciones de información. ${ }^{11}$

A continuación se presentan matrices comparativas sobre la formación de profesionales de la información en Venezuela en dependencia del nivel académico: Licenciatura, estudios de postgrado y estudios técnicos. Estas matrices nos permitirán relacionar aspectos como la duración de los estudios en años, así como, su distribución bien sea en semestres, trimestres o trayectos anuales. También, se estiman las unidades de crédito necesarias para la titulación y, por último, la visión epistemológica. Este último elemento, nos permitirá conocer la orientación en temática de los planes de estudios que gestionan las instituciones responsables de la formación, la influencia que pudiera existir sobre el abordaje de los temas inherentes a la formación de profesionales de la información (véase Cuadro 2).

\begin{tabular}{|c|c|c|c|c|}
\hline $\begin{array}{l}\text { Institución } \\
\text { Responsable }\end{array}$ & Titulación & $\begin{array}{c}\text { Duración en } \\
\text { años }\end{array}$ & $\begin{array}{l}\text { Unidades } \\
\text { de Crédito }\end{array}$ & Visión Epistemológica \\
\hline $\begin{array}{l}\text { Universidad } \\
\text { Central de } \\
\text { Venezuela }\end{array}$ & $\begin{array}{l}\text { *Licenciado en } \\
\text { Bibliotecología } \\
\text { ^Licenciado en } \\
\text { Archivología }\end{array}$ & $\begin{array}{l}5 \text { años, } \\
\text { distribuidos en } \\
\text { semestres. }\end{array}$ & 189 U.C. & $\begin{array}{l}\text { Visión disciplinar con énfasis en los } \\
\text { aspectos técnico-prácticos de las } \\
\text { actividades bibliotecaria y archivística. }\end{array}$ \\
\hline $\begin{array}{l}\text { Universidad del } \\
\text { Zulia }\end{array}$ & $\begin{array}{l}\text { *Licenciado en } \\
\text { Bibliotecología y } \\
\text { Archivología }\end{array}$ & $\begin{array}{l}5 \text { años, } \\
\text { distribuidos en } \\
\text { semestres. }\end{array}$ & 186 U.C. & $\begin{array}{l}\text { Visión disciplinar con énfasis en los } \\
\text { aspectos técnico-prácticos de las } \\
\text { actividades bibliotecaria y archivística } \\
\text { con basamento holístico. }\end{array}$ \\
\hline $\begin{array}{l}\text { Universidad } \\
\text { Politécnica } \\
\text { Territorial de Lara } \\
\text { Andrés Eloy Blanco }\end{array}$ & $\begin{array}{l}\text { *Licenciado en } \\
\text { Ciencias de la } \\
\text { Información }\end{array}$ & $\begin{array}{l}4 \text { años, } \\
\text { distribuidos en } \\
\text { trayectos y } \\
\text { periodos. }\end{array}$ & 187 U.C. & $\begin{array}{l}\text { Declaratoria interdisciplinaria a nivel del } \\
\text { diseño curricular. }\end{array}$ \\
\hline $\begin{array}{l}\text { Universidad } \\
\text { Yacambú }\end{array}$ & $\begin{array}{l}\text { *Licenciado en } \\
\text { Información y } \\
\text { Documentación }\end{array}$ & $\begin{array}{l}4 \text { o } 5 \text { años en } \\
\text { dependencia } \\
\text { de la } \\
\text { modalidad, } \\
\text { distribución en } \\
\text { trimestres. }\end{array}$ & 200 U.C. & $\begin{array}{l}\text { Visión disciplinar con énfasis en los } \\
\text { aspectos técnico-prácticos de la } \\
\text { actividad documental y las tecnologías } \\
\text { de información digital. }\end{array}$ \\
\hline
\end{tabular}

Cuadro 2: Matriz comparativa de la formación del profesional de la información a nivel de licenciatura.

Gracias a esta matriz comparativa, es posible advertir que la formación a nivel de licenciatura oscila entre los cuatro y cinco años. En el caso de las universidades Central de Venezuela y del Zulia la duración de los estudios es de cinco años y su distribución es semestral con régimen de prelaciones tal y como lo establecen los reglamentos internos de las mencionadas casas de estudio. La Universidad Politécnica Territorial de Lara Andrés Eloy Blanco prevé la formación en cuatro años que denomina trayectos y estos a su vez se dividen en dos periodos cada uno. La Universidad Yacambú, por su parte, prevé la formación en cuatro o cinco años en dependencia de la modalidad bien sea esta presencial (culminación en cuatro años) o a distancia (culminación en cinco años) para ambas modalidades la distribución es trimestral.

En lo referido a las unidades de crédito necesarias para la titulación las instituciones universitarias revisadas conforman planes de estudio con una oscilación que va de ciento ochenta y seis unidades de crédito, caso de la Universidad del Zulia, y doscientas unidades de crédito, caso de la Universidad Yacambú. Este hecho se ajusta a los lineamientos dispuestos por el Consejo Nacional de Universidades, órgano dependiente del Ministerio del Poder Popular para la Educación Universitaria, en materia de carga crediticia. También, resalta el hecho que, a excepción de la Universidad Politécnica Territorial de Lara Andrés Eloy Blanco, las restantes prevén la entrega de un trabajo especial de grado para la culminación de la formación universitaria. Esta excepción es el resultado 
de la incorporación, por parte de la universidad politécnica, del Proyecto Socio-Integrador como elemento transversal en el diseño curricular.

En lo referido a la visión epistemológica predominante, se observa que las universidades Central de Venezuela, del Zulia y Yacambú se orientan bajo criterios disciplinares, es decir, la visión de las denominadas Ciencias de la Información es una yuxtaposición de disciplinas científicas, cada una con independencia epistémica, objetos de estudios diferenciados y procesos técnicos de la información separados en dependencia de la actividad operativa sin ninguna búsqueda de la convergencia disciplinar. Aún cuando, la Universidad del Zulia confiere el título en Bibliotecología y Archivología unitariamente y declaran la visión holística del currículo, el abordaje se realiza disciplinarmente. También, resalta el hecho de la importancia que se confiere a los aspectos relacionados con las actividades técnico-prácticas del procesamiento de la información y en las tecnologías de la información digital. Este último aspecto está explicito en los planes de estudio de la Universidad Yacambú. ${ }^{9}$

La Universidad Politécnica Territorial de Lara Andrés Eloy Blanco ha conformado un diseño curricular que declara explícitamente el abordaje interdisciplinario como basamento teórico-curricular. Es la única universidad venezolana que confiere el título en Ciencias de la Información, este hecho resulta inédito, toda vez que es el primer diseño curricular en Venezuela que declara la interdisciplinariedad como basamento del abordaje, sin embargo, esto en la práctica ha sido difícil de concretar incluso en el plan de estudios la disciplinariedad se hace manifiesta en los sinópticos temáticos de las unidades curriculares o asignaturas. ${ }^{7}$

El Comité Interinstitucional del Programa Nacional de Formación en Ciencias de la Información, consciente de la situación antes planteada, intenta el rediseño del plan de estudios y en ese sentido serán reorganizadas las unidades curriculares no sólo en su denominación sino también en la estructuración temática. Estos cambios están, a la fecha, en proceso de realización.

Seguidamente, se presenta la matriz comparativa acerca de la formación del profesional de la información en Venezuela a nivel de maestría. Esta matriz presenta además de la institución universitaria responsable de la administración de la oferta académica, la titulación que se confiere, las unidades de crédito que conforman la oferta y la visión epistemológica dominante (Cuadro 3).

\begin{tabular}{|c|c|c|c|}
\hline $\begin{array}{l}\text { Institución } \\
\text { Responsable }\end{array}$ & Titulación & $\begin{array}{l}\text { Unidades de } \\
\text { Crédito }\end{array}$ & Visión Epistemológica \\
\hline $\begin{array}{l}\text { Universidad Central } \\
\text { de Venezuela }\end{array}$ & $\begin{array}{l}\text { Maestría en Información } \\
\text { y Comunicación para el } \\
\text { Desarrollo }\end{array}$ & 40 U.C. & Tecnologicista \\
\hline Universidad del Zulia & $\begin{array}{l}\text { Maestría en Ciencias de } \\
\text { la Información Mención } \\
\text { Gerencia del } \\
\text { Conocimiento }\end{array}$ & 39 U.C. & Gerencial \\
\hline $\begin{array}{l}\text { Universidad Nacional } \\
\text { Experimental } \\
\text { Francisco de } \\
\text { Miranda }\end{array}$ & Maestría en Museología & 36 U.C. & $\begin{array}{l}\text { Disciplinar sin la presencia de elementos } \\
\text { relacionados con la documentación } \\
\text { museística y sus importancia en la } \\
\text { dinámica de los museos. }\end{array}$ \\
\hline $\begin{array}{l}\text { Universidad José } \\
\text { Antonio Páez }\end{array}$ & $\begin{array}{l}\text { Maestría en Gerencia de } \\
\text { Tecnologías de la } \\
\text { Información }\end{array}$ & 32 U.C. & Gerencial-Tecnologicista \\
\hline
\end{tabular}

Cuadro 3: Matriz comparativa formación del profesional de la información a nivel de maestría

Como es posible advertir, la formación de profesionales de la información a nivel de maestría en Venezuela se lleva a cabo en cuatro universidades, tres de las cuales son públicas y una privada, sin embargo, los estudios de cuarto nivel en las universidades autónomas no son gratuitos, es decir, los interesados deben cancelar el costo total de los estudios de maestría. En lo referido a la titulación, cada maestría posee una denominación diferente y solamente la Universidad del Zulia la denomina Maestría en Ciencias de la Información; es la única que agrega a la titulación una mención en este caso Gerencia del Conocimiento. En lo referente a la carga crediticia, las cuatro ofertas se ajustan a los lineamientos del Consejo Nacional de Universidades y oscilan entre las treinta y dos y cuarenta unidades de crédito. En lo que respecta a la visión epistemológica, una revisión de los planes de 
estudio da cuenta del enfoque predominante: en el caso de la maestría denominada Información y Comunicación para el Desarrollo, la cual es administrada por la Universidad Central de Venezuela, su enfoque es principalmente tecnológico, es decir, focaliza los ejes temáticos en las tecnologías de la información digital; lo mismo sucede con la maestría administrada por la Universidad José Antonio Páez, ésta sin embargo, complementa las temáticas abordadas con componentes gerenciales. La Universidad del Zulia, por su parte, focaliza sus esfuerzos temáticos en aspectos meramente gerenciales. La Universidad Nacional Experimental Francisco de Miranda, por su parte, centra sus esfuerzos en los aspectos museológicos y museográficos, es decir, en la valoración del objeto museable desde la perspectiva estética y como evidencia de un hecho histórico sin que la documentación museológica y su importancia en la agregación de valor por la vía informacional a la discursiva expositiva.

Seguidamente presentamos una matriz comparativa de la formación informacional a nivel de técnico medio y diplomados (véase Cuadro 4).

\begin{tabular}{|c|c|c|c|}
\hline $\begin{array}{l}\text { Institución } \\
\text { Responsable }\end{array}$ & Titulación & $\begin{array}{l}\text { Duración en } \\
\text { horas } \\
\text { académicas }\end{array}$ & Objetivos \\
\hline \multirow{2}{*}{$\begin{array}{l}\text { Universidad de } \\
\text { Carabobo }\end{array}$} & $\begin{array}{l}{ }^{*} \text { Analista } \\
\text { Documentalista. }\end{array}$ & 164 & $\begin{array}{l}\text { Formar recursos humanos con competencias para el } \\
\text { manejo de la información y documentación en tareas } \\
\text { especificas en el procesamiento, evaluación y análisis de } \\
\text { documentos científicos, depositados en bibliotecas, } \\
\text { centros de información e investigación, pertenecientes a } \\
\text { dependencias universitarias e instituciones privadas de } \\
\text { la región y de otras localidades. }\end{array}$ \\
\hline & $\begin{array}{l}\text { *Asistente de } \\
\text { Biblioteca. }\end{array}$ & 100 & $\begin{array}{l}\text { Formar recursos humanos con competencias para asistir } \\
\text { a los profesionales en el manejo de la información y } \\
\text { documentación en tareas especificas de procesamiento y } \\
\text { búsqueda de documentos depositados en Bibliotecas, } \\
\text { centros de información e investigación, pertenecientes a } \\
\text { instituciones publicas y privadas de la región. }\end{array}$ \\
\hline \multirow{2}{*}{$\begin{array}{l}\text { Universidad } \\
\text { Pedagógica } \\
\text { Experimental } \\
\text { Libertador }\end{array}$} & $\begin{array}{l}\text { *Diplomado en } \\
\text { Bibliotecas } \\
\text { Escolares. }\end{array}$ & 200 & \multirow{2}{*}{$\begin{array}{l}\text { Formar docentes en ejercicio es aspectos relacionados } \\
\text { con la actividad bibliotecaria, bien sea, para el } \\
\text { desempeño en bibliotecas escolares o en unidades de } \\
\text { información dependientes de centros de investigación } \\
\text { adscritas al Ministerio del Poder Popular para la } \\
\text { Educación. }\end{array}$} \\
\hline & $\begin{array}{l}\text { *Diplomado en } \\
\text { Bibliotecología y } \\
\text { Ciencias de la } \\
\text { Información. }\end{array}$ & 200 & \\
\hline
\end{tabular}

Cuadro 4: Matriz comparativa formación del profesional de la información a nivel de cursos técnicos y diplomados

La matriz comparativa sobre la formación de profesionales de la información a nivel de técnico medio y diplomado, nos presenta cuatro ofertas relacionadas con la dinámica informacional, cabe destacar que estas cuatro ofertas académicas están presentes en dos universidades ubicadas en la zona central del país, estados Aragua y Carabobo. Las ofertas académicas que presenta la Universidad de Carabobo están dirigidas al público en general y son accesibles a través de FUNDACID, unidad dependiente de la Dirección de Biblioteca Central de la universidad.

En el caso de los diplomados que oferta la Universidad Pedagógica Experimental Libertador a través del Instituto Pedagógico Rural El Mácaro, están dirigidos a docentes en ejercicio interesados en incursionar en la actividad bibliotecaria, bien a nivel escolar como en unidades de investigación dependientes del Ministerio del Poder Popular para la Educación

En los cuatro casos es posible advertir que los ejes temáticos se orientan hacia los aspectos de catalogación y clasificación documental, destacando el Diplomado en Bibliotecología y Ciencias de la Información que incorpora elementos temáticos como la biblioestadística, la formación de usuarios y las bibliotecas digitales. 


\section{Conclusiones}

Una vez analizados, algunos elementos del contexto histórico local previo a la instauración de programas académicos para la formación de profesionales de la información en Venezuela, su proceso evolutivo, las titulaciones conferidas, algunos elementos tecno-curriculares y la visión epistemológica para el abordaje temático de los planes de estudio vigentes en las universidades venezolanas, podemos concluir:

La formación académica de profesionales de la información en Venezuela se inició en 1948 con la implantación de los estudios técnicos en Biblioteconomía en la Universidad Central de Venezuela, después de un proceso evolutivo interno en esa casa de estudios se erige la Escuela de Bibliotecología y Archivología y se adscribe a la Facultad de Humanidades y Educación y se instaura en la década de los sesenta del siglo XX los estudios a nivel de licenciatura.

En esta misma década, más precisamente en el año de 1962, la Universidad del Zulia, ubicada al occidente del país, inicia la formación de profesionales de la información con la creación de los estudios en Bibliotecología y Archivología en la Escuela de Letras. A partir del año de 1983, se crea la Escuela de Bibliotecología y Archivología y se adscribe a la Facultad de Humanidades y Educación.

Durante casi cincuenta años la formación de profesionales de la información fue tarea exclusiva de las universidades Central de Venezuela y del Zulia, hasta que en 1997 se crea una oferta académica a nivel técnicouniversitario en el Instituto Universitario Experimental de Tecnología Andrés Eloy Blanco, ubicado en al ciudad de Barquisimeto en el Estado Lara. Después de completar un ciclo que permitió alcanzar entre otros elementos, madurez curricular de la oferta académica denominada Técnico Superior Universitario en Información y Documentación, así como, emprender la trasformación institucional se crea el Programa Nacional de Formación en Ciencias de la Información y se convierte el instituto tecnológico en la Universidad Politécnica Territorial de Lara Andrés Eloy Blanco.

También en el Estado Lara, específicamente en la Universidad Yacambú, institución de carácter privado se creó en la década de los noventa la licenciatura en Información y Documentación, la cual hasta la fecha sigue ingresando estudiantes interesados en recibir formación profesional en el campo informacional.

En lo referido a estudios de postgrado y a formación técnica a nivel medio, destacan las iniciativas de las universidades Central de Venezuela, del Zulia y José Antonio Páez, las cuales ofertan maestrías relacionadas con el campo informacional desde la perspectiva documental. Así mismo, las universidades de Carabobo y Pedagógica Experimental Libertador ofertan cursos a nivel de técnico medio y diplomados, todos relacionado con aspectos inherentes a la actividad bibliotecaria.

\section{Bibliografía}

1. Himiob,S. (2008). Historia de la Biblioteca Nacional de Venezuela. Caracas: Instituto Autónomo Biblioteca Nacional y de Servicios de Bibliotecas.

2. Mastromatteo, E. (2005). Bases, Fundamentos y Perfil Profesional: Aportes para el Cambio Curricular de la EBA-UCV. [tesis] Universidad Central de Venezuela.

3. Facultad de Humanidades y Educación de la Universidad del Zulia [página web]. Bibliotecología y Archivología: Reseña Histórica [actualizado 2013; citado 23 Abril 2013]. Disponible en: http://www.fhe.luz.edu.ve/index.php?option=com content\&task=view\&id=577\&\&ltemid=242

4. Pirela, J. (1998). Desarrollo Curricular de la Escuela de Bibliotecología y Archivología de la Universidad del Zulia. V Encuentro de Educadores e Investigadores de Bibliotecología, Archivología y Ciencia de la Información de Iberoamérica y el Caribe. Maracaibo: Universidad del Zulia.

5. Facultad de Humanidades y Educación de la Universidad del Zulia [página web]. Escuela de Bibliotecología y Archivología: Mapa Curricular [actualizado 2013; citado 23 Abril 2013 ].

Disponible en:

http://www.fhe.luz.edu.ve/index.php?option=com content\&task=view\&id=581\&ltemid=242 
6. Instituto Universitario Experimental de Tecnología Andrés Eloy Blanco. (1993). Proyecto de Creación de la Carrera Técnico Superior en Información y Documentación: Diseño Curricular. Barquisimeto: IUETAEB.

7. Instituto Universitario Experimental de Tecnología Andrés Eloy Blanco. (2008). Programa Nacional de Formación en Ciencias de la Información: Diseño Curricular. Barquisimeto: IUETAEB.

8. Universidad Nacional Experimental Francisco de Miranda [página web]. Estudios de Postgrado [actualizado 2013; citado 8 Marzo 2013]. Disponible en: http://www.unefm.edu.ve

9. Universidad Yacambú [página web]. Diseño Curricular Licenciatura en Información y Documentación[actualizado 2011; citado 9 Marzo 2013]. Disponible en: http://www.uny.edu.ve/index.php/facultades/humanidades/lic-en-informacion-y-documentacion

10. Universidad José Antonio Páez [página web]. Estudios de Postgrado: Maestría en Gerencia de Tecnología de la Información [actualizado 2013; citado 12 Marzo 2013]. Disponible en: http://www.ujap.edu.ve/Universitas/html/principal/index.htm

11. Fundación Centro de Información y Documentación Universidad de Carabobo [página web]. Cursos [actualizado 2013; citado 19 Abril 2013 ]Disponible en: http://www.cid.uc.edu.ve/?q=listacyd

12. Instituto Pedagógico Rural El Mácaro [página web]. Diplomados [actualizado 2012; citado 22 de Abril 2013]. Disponible en: http://www.iprm.upel.edu.ve

13. Universidad Simón Bolívar Decanato de Postgrado [página web]. Orígenes [actualizado 2013; citado 10 Marzo 2013]. Disponible en: http://www.postgrado.usb.ve/organizacion/origenes

14. Tribunal Supremo de Justicia de la República Bolivariana de Venezuela [base de datos en Internet]. Gaceta Oficial №39.593 del 13/1/2011: Resolución mediante la cual se reconoce los estudios conducentes al Título de Bibliotecología y Ciencias de la Información, conferido por la Universidad de La Habana, en la ciudad de La Habana, República de Cuba, a los ciudadanos y ciudadanas que en ella se mencionan - [citado 15 Abril 2013]. Disponible en: http://www.tsj.gov.ve/gaceta/gacetaoficial.asp Actualización diaria. 


\section{Datos del autor}

\section{Juan Carlos Linárez Pérez}

Licenciado en Bibliotecología y Ciencia de la Información por la Universidad de La Habana (Cuba). Técnico Superior Universitario en Información y Documentación. Departamento de Información y Documentación. Instituto Universitario Experimental de Tecnología "Andrés Eloy Blanco" (Barquisimeto, Venezuela). Actualmente se desenvuelve como Coordinador General del Programa de Fomento de la Educación Universitaria a Distancia Mediadas por Tecnologías de la Información y la Comunicación, perteneciente a la Oficina de Planificación del Sector Universitario - OPSU (Venezuela).

juanklinarez@yahoo.com

Recibido - Received : 2014-01-30

Aceptado - Accepted: 2015-03-30

\section{(cc) $\mathrm{Br}$}

This work is licensed under a Creative Commons Attribution 4.0

United States License.

\section{ULIS D-Senter}

This journal is published by the University Library System of the University of Pittsburgh as part of its D-Scribe Digital Publishing Program and is cosponsored by the University of Pittsburgh Press. 